\section{Symptomatische Schlafstörungen}

\section{Helga Peter}

Marburg, Deutschland

\section{Synonyme}

Sekundäre Schlafstörungen

\section{Englischer Begriff}

symptomatic sleep disorders; secondary sleep disorders

\section{Definition}

Bei den symptomatischen Schlafstörungen steht die Symptomatik von \ „Insomnie“, \ „Tagesschläfrigkeit“ bzw. einem nicht erholsamen Schlaf im Vordergrund des Beschwerdebildes einer vorbestehenden oder im Ausbruch befindlichen psychiatrischen, neurologischen oder anderen körperlichen Grunderkrankung. Bei manchen psychiatrischen Erkrankungen wie \ "Affektive Störungen“ oder \ „Angststörungen“ können die Schlafstörungen oftmals dem Ausbruch der zugrunde liegenden psychiatrischen Erkrankung deutlich vorausgehen, und sie können auch nach der Entwicklung des Vollbildes der Erkrankung noch das subjektive Beschwerdebild der Patienten beherrschen. Symptomatische Schlafstö- rungen bei psychiatrischen, neurologischen oder anderen körperlichen Erkrankungen ziehen häufig spezifische diagnostische oder therapeutische Konsequenzen nach sich.

Die Internationale Klassifikation der Schlafstörungen hatte die symptomatischen Schlafstörungen in der ICSD-R von 1997 als gleichberechtigte Hauptdiagnosegruppe „Schlafstörungen bei anderen Erkrankungen" geführt, neben den Hauptdiagnosegruppen Dyssomnien, Zirkadiane Rhythmusstörungen und Parasomnien. Die ICSD-2 (2005) war um Kompatibilität mit dem Regelwerk der allgemeingültigen Internationalen Klassifikation der Krankheiten (ICD) bemüht. Das verlangte, dass an erster Stelle die jeweils einer Symptomatik zugrunde liegende Erkrankung zu kodieren war. Damit entfielen in der ICSD-2 die symptomatischen Schlafstörungen als kodierfähige Hauptkategorie. Es sollte vielmehr die betreffende psychiatrische, neurologische oder andere körperliche Erkrankung gemäß dem allgemeinen Diagnoseschlüssel ICD kodiert werden, und zusätzlich sollte eine Kodierung entsprechend der Art der symptomatischen Schlafstörung als Insomnie, Hypersomnie, Zirkadiane Rhythmusstörung, Schlafbezogene Bewegungsstörung oder Parasomnie erfolgen; sie trug dann den Zusatz „durch andere Erkrankung" (due to medical condition) oder ,durch Medikamente oder Substanzen" (due to drug or substance). Allein bei den Insomnien gab es zusätzlich die Diagnose „Insomnie durch psychiatrische Erkrankung“ (insomnia due to mental disorder). Dieses Vorgehen wird im Prinzip in der ICSD-3 beibehalten, bei den einzelnen Diagnosegruppen haben sich jedoch Änderungen gegenüber der ICSD-2 ergeben. Zum Vergleich der beiden Versionen siehe $>$ „ICSD-3“. 